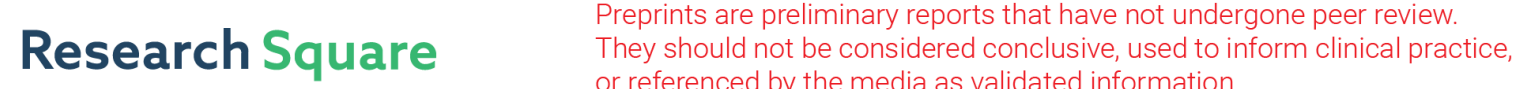 or referenced by the media as validated information. \\ Three dimensional acoustic tweezers with acoustic vortex streaming
}

\section{Junfei Li}

Duke University https://orcid.org/0000-0002-5184-1737

\section{Alexandru Crivoi}

Nanyang Technological University

\section{Xiuyuan Peng}

Duke University

\section{Lu Shen}

Nanyang Technological University

\section{Yunjiao Pu}

Duke University

\section{Zheng Fan}

Nanyang Technological University https://orcid.org/0000-0002-7818-153X

Steven Cummer ( $\nabla$ cummer@duke.edu )

Duke University

\section{Article}

Keywords: fluid dynamics, 3D acoustic tweezers, acoustic vortex streaming

Posted Date: February 17th, 2021

DOI: https://doi.org/10.21203/rs.3.rs-236078/v1

License: (c) (1) This work is licensed under a Creative Commons Attribution 4.0 International License. Read Full License

Version of Record: A version of this preprint was published at Communications Physics on June 3rd, 2021. See the published version at https://doi.org/10.1038/s42005-021-00617-0. 


\title{
Three dimensional acoustic tweezers with acoustic vortex streaming
}

\author{
Junfei $\mathrm{Li}^{1, \star}$, Alexandru Crivoi ${ }^{2, \star}$, Xiuyuan Peng ${ }^{1}$, Lu Shen ${ }^{2}$, Yunjiao $\mathrm{Pu}^{1}$ Zheng Fan ${ }^{2}$, and Steven A. Cummer ${ }^{1}$ \\ ${ }^{1}$ Department of Electrical and Computer Engineering, \\ Duke University, Durham, North Carolina 27708, USA \\ ${ }^{2}$ School of Mechanical and Aerospace Engineering, Nanyang Technological University, \\ 50 Nanyang Avenue, Singapore, 639798, Singapore \\ * J.L. and A.C. contributed equally to this work. Correspondence and requests for materials \\ should be addressed to Z.F. (zfan@ntu.edu.sg) or to S.A.C. (cummer@ee.duke.edu)
}

\begin{abstract}
Acoustic tweezers use ultrasound for contact-free manipulation of particles from millimeter to sub-micrometer scale. Particle trapping originated in either radiation forces or acoustic streaming fields. Acoustic tweezers based on single-beam focused acoustic vortices have attracted considerable attention due to their selective trapping capability, but have proven difficult to use for 3D trapping without a complex transducer array and significant constraints on the trapped particle properties. Here we demonstrate the first 3D acoustic tweezer that uses a single transducer and combines the radiation force for trapping in two dimensions with the streaming force to provide levitation in the third dimension. The idea is demonstrated in both simulation and experiments, and the achieved levitation force reaches three orders of magnitude larger than for previous 3D trapping. This hybrid acoustic tweezer that integrates acoustic streaming adds a new twist to the approach and expands the range of particles that can be manipulated.
\end{abstract}

\section{Introduction}

Precise and contact-free manipulation of physical and biological objects is highly desirable in a wide range of fields that include nanofabrication, micro- and nano-robotics, drug delivery, and cell and tissue engineering. To this end, acoustic tweezers serve as a fast-developing platform for precise manipulation across a broad object size range $[1,2]$. There are two primary types of acoustic tweezers under development at present: radiation force tweezers and acoustic-streaming tweezers.

Radiation force tweezers, in which the acoustic radiation force acts as the trap, can be divided into standing-wave tweezers and traveling-wave tweezers. To date, most demonstrated acoustic tweezers are standing wave tweezers that use counter-propagating waves to create a mesh of standing-wave nodes and antinodes where the particles are trapped [3-14]. Such systems are particularly suitable for manipulating groups of particles, but the chessboard-like node network precludes object selectivity. In addition, standing wave trapping typically requires multiple transducers that surround the trapping region, which adds complexity and makes it incompatible with some application scenarios, especially those that involve fixed object inside the trapping region.

Travelling-wave acoustic tweezers, in contrast, form acoustic pressure nodes by designing the structure of a single beam instead of using interference between beams. They are typically achieved by controlling the phase patterns across the radiation aperture [15-21]. Several structured beams are proposed to date. Particularly, strong localization and creation of acoustic pressure node can be simultaneously fulfilled by imparting angular momentum into the field and generating what are known as acoustic vortices [16, 22-28]. With recent developments in the theory of acoustic radiation force, acoustic tweezing with vortices has been experimentally demonstrated [23, 28-31].

However, acoustic vortices achieved with either cylindrical or spherical harmonics create a node line, rather than a point, along the axial direction, limiting its ability to trap particles in 3D. The ability to obtain a 3D trap and to pick up one particle independently of its neighbors using acoustic vortex was only demonstrated recently by Baresch et al. [31]. The three-dimensional trapping force is achieved by the dipolar mode on the propagation axis, which sets limitations on the particle parameters and adds complexity in field shape control. Also, the vertical force provided by radiation force is orders of magnitude smaller than lateral forces, making it hard to handle large and heavy particles. For large particles, it is necessary to lower the frequency that makes the trap weaker, and the acoustic power must be increased to achieve larger force. But nonlinear perturbations, such as acoustic streaming, will inevitably appear and start to destroy this type of trap. In addition, radiation forces and gravity does not scale linearly with particle size and operating frequency, so that an established travelling wave tweezer may fail when it is scaled to fit a wider range of particle sizes. For a fixed tweezer, the radiation force $\left(F_{r} \propto R^{3}\right)$ decreases faster for small particles than the drag $\left(F_{d} \propto R\right)$ initiated by acoustic streaming, also restraining the size range of the particles that can be manipulated. Most approaches have used transducer arrays that are expensive and complex.

Both standing-wave tweezers and traveling-wave tweezers rely on acoustic radiation force to directly manipulate 
particles, whereas acoustic-streaming tweezers take advantage of the nonlinear Rayleigh streaming induced fluid flows [32], and thus handle particles indirectly in fluids by creating streaming vortices [33] with oscillating bubbles [34] or rigid structures $[35,36]$. These devices tend to be simple devices that are easy to operate, but offer low degree of spatial resolution, because microbubble and microstructure-based phenomena are nonlinear and difficult to control [2]. Fluid manipulation has been demonstrated using controlled pumping [37], but is limited to 2D, and requires sophisticated control over the source array.

Here we propose a hybrid 3D single beam acoustic tweezer by combining the radiation force and acoustic streaming. We exploit the Eckart streaming [32] and demonstrate that, instead of being a nuisance, carefully designed acoustic streaming can be embedded in the focused acoustic vortex to create a fully 3D trap. As a proof of concept, we generated a focused acoustic vortex with a single piezoelectric transducer and a passive polydimethylsiloxane (PDMS) lens. The experimental levitation force provided by streaming reaches 3 orders magnitude larger than previously reported [31], and allows a wider range of particle size, shape and material properties. We demonstrate this three-dimensional acoustic tweezer first by simulation and experimental measurement of the acoustic field. Then the acoustic streaming flow field is measured with particle image velocimetry (PIV). Finally, levitation, trapping and 3D manipulation of a particle is demonstrated in a fluid environment.

\section{Results}

\section{Lens for converging acoustic vortex}

An acoustic vortex produces an acoustic node line along the axial direction, therefore, it serves as ideal candidate for a 2D acoustic trap. Focusing an acoustic vortex not only increases its spatial selectivity, but also makes the $2 \mathrm{D}$ trap stronger. 3D trapping with radiation force in a focused acoustic vortex requires careful selection of material properties and sizes, and yet the achieved axial force is several orders magnitude weaker than lateral forces. Moreover, focused ultrasound in liquids induces nonlinear streaming, especially when lifting heavier particles that require higher wave amplitude. Such streaming can easily disrupt acoustic traps based on radiation forces [31]. However, a focused acoustic field will also induce a streaming flow localized around its focal point. The steaming velocity reaches its maximum above the focal point, and then decreases after that. Such localized, steady gradient flow velocity along the axis, if controlled properly, can also provide the gradient lifting force against gravity to create a 3D trap, as is shown in Fig. 1(a). Such a mechanism offers three advantages: i) it doesn't require resonance modes of the particle to provide lifting force along $z$ axis, as the levitation is provided by the drag in the steady flow, so it sets less constraints on particle size and materials; ii) the steady flow velocity can reach several centimeters per second, especially suitable for large and heavy particles that cannot be handled with radiation forces; and iii) the drag force and trapping position can be tuned by controlling the streaming flow velocity. Controlling this streaming force is our strategy for creating a $3 \mathrm{D}$ trap.

As a demonstration, the focused vortex field is generated by placing a PDMS lens on a circular lead zirconate titanate (PZT) transducer $38 \mathrm{~mm}$ in diameter. The lens design principle is based on the combination of approaches previously developed for the acoustic holograms and holographic elements[16, 17]. First, the required 2D phase map of the acoustic wave on the source plane just above the lens is calculated. This phase map has a specific signature pattern in order to achieve the focused vortex field on the target plane after the wave propagation in the $z$-direction. It was previously calculated by Marzo et al. [16] using the Broyden-Fletcher-Goldfarb-Shanno (BFGS) optimization method that the optimal source plane phase field producing the focused vortex trap is a direct sum of the (i) focus lens and (ii) simple vortex phase signatures. Therefore, the phase field at the exit of the PDMS lens structure is calculated analytically for each pixel of the source plane as a sum of the Fresnel lens phase and the simple vortex phase at this pixel location. The acoustic wave source amplitude is assumed to be constant here for simplicity, as the PDMS has low attenuation $(0.1352 \mathrm{~dB} / \mathrm{mm})$, and has an impedance value close to water. Consequently, the $2 \mathrm{D}$ thickness map of the PDMS lens is calculated from the corresponding 2D phase delay map based on the sound wavelength difference in PDMS material and water (see Methods for details). The resulting 3D shape of the lens is shown in Figure 1 (a) and has the height contour lines following the Fermat-Archimedes spiral branches.

\section{Simulation of the acoustic field and streaming field}

The finite element simulations of the acoustic wave propagation were performed in the frequency domain using the open-source finite element solver Code Aster [38]. The simulation domain is a 3D waveguide where the bottom wall represents the wave source and the normal axis $(z)$ is the propagation direction. The analytically calculated phase field at the source plane was used as a boundary condition for the bottom plane $(z=0)$. The side walls were treated 
as hard wall boundaries, and the anechoic exit condition was assigned to the top boundary plane. The weak form of the Helmholtz equation is numerically solved by Code Aster in the single-phase acoustics settings, and the acoustic intensity and complex pressure amplitude is calculated in the entire 3D simulation domain.

The results in the $O x z$ sectional plane represent the development of the vortex trap pattern in the propagation direction with the maximal amplitude observed near the focal position (see Figure 1(b)). The phase signature in the center part of the focal plane indicates the orbital angular momentum characteristic for the focused vortex beams. Figure 2 (b-i) shows the numerical and experimental results in two sectional planes: the Fxy focal plane and the $O x z$ central section of the box. The intensity and phase field structure in the focal plane matches the signature of the focused vortex trap described in previous works [17]. The finite element results show good agreement with their experimental counterparts.

The acoustic streaming flow was simulated using another open-source tool, OpenFOAM [39]. The streaming effect modelling was performed in three stages as previously suggested in [40]: (i) simulation of the wave propagation in time domain using the compressible flow CFD solver, (ii) time-averaging of the effective non-linear equation term to calculate the body force driving the acoustic streaming flow, and (iii) using the incompressible steady-state CFD solver to calculate the streaming velocity field by adding the effective external force equation term calculated in step (ii). All the required solvers are included in the default OpenFOAM distribution with minor additional code modifications required.

The results of the streaming fluid flow simulation are presented in Figure 3. Figures 3 (b-c) show the streaming velocity magnitude distribution in the focal plane $(z=30 \mathrm{~mm})$ and the sectional plane in the sound wave propagation direction $(O x z)$. The results in the $O x z$ plane show a significant outward fluid flow away from the sound source near the focal point (Figure $3(\mathrm{c})$ ). The flow converges and increases in magnitude towards the focal plane and carries simulated particles from the periphery of acoustic lens region towards the axis of symmetry. The focal plane section results indicate that the simulated flow magnitude is actually weaker along the axis itself but reaches the maximum in the surrounding cylindrical region, forming a fluid vortex where the acoustic vortex is located (Figure 3 (b)). These combined effects will not only trap the particle in the $x-y$ plane, but provide a strong localized drag flow for levitation and thus trapping in the $z$ direction.

\section{Experimental demonstration of 3D trapping}

Measurements confirm that the design is capable of 3D particle trapping can be used to move a particle along a prescribed trajectory in three dimensions. The lens in the experiment was fabricated with (PDMS) molding. It is then attached to a PZT $38 \mathrm{~mm}$ in diameter and $4.1 \mathrm{~mm}$ in thickness, with a $500 \mathrm{kHz}$ resonance frequency. The acoustic field created by the lens is first measured by a hydrophone attached to a 3D positioning stage, as illustrated in Fig. 2(a). The scanned acoustic pressure and phase profile across $x-y$ plane and $x-z$ plane in Figure 2(c, e, g, i) shows good agreement with the corresponding simulations. The discrepancy between simulation and experiment can be attributed to the slight error in the phase profile caused by fabrication error, slight change of sound speed in water due to the impurity, and shear modulus in PDMS. The acoustic node along the $\mathrm{z}$ axis where the particles can be trapped is clearly seen. Compared with a cylindrical vortex that generates a non-negligible secondary ring, the spatial selectivity of such a focused vortex is greatly enhanced [23, 28].

The acoustic streaming field is measured with particle image velocimetry (PIV). The experimental setup is illustrated in Fig. 3(a). Polyamide seeding particles with mean size $60 \mu \mathrm{m}$ are dispersed in the de-ionized water. A fan-shaped $532 \mathrm{~nm}$ laser beam is aligned with $\mathrm{x}-\mathrm{z}$ plane. Light scattered by the seeding particles is then captured and recorded by a camera, therefore the flow field can be indirectly measured by tracking the particles using an ensemble correlation PIV algorithm. The results in Fig. 3(d) show that the upward flow converges to the focal point, reaching maximum velocity at the focused region, and diverges after passing the focal plane. The drag force provided by such a localized steady flow as the serves as levitating force and enables the 3D trapping and manipulation.

To demonstrate the 3D trapping capability of the proposed acoustic tweezer, we first demonstrate suspending a cellulose acetate polymer sphere as shown in Fig. 4(a) and mov. S1. The particle has a diameter $1.5 \mathrm{~mm}$ with density $1.3 \mathrm{~g} / \mathrm{cm}^{3}$. The source is driven by an RMS voltage $42 \mathrm{~V}$. The particle is levitated at $z=42 \mathrm{~mm}$. The calculated levitation force provided by the tweezer reaches $5.2 \mu \mathrm{N}$, which is 3 orders of magnitude larger than the previously reported tweezer that relies on radiation force [31]. The radiation force field in our case is calculated semi-analytically, and the lateral force along the center line in the trapping plane is shown in Fig. 4(b), while the radiation force along the $z$ axis is shown in Fig. 4(c). The red dots denote the trapping position. We can see at the trapping position, the radiation force $(0.02 \mu \mathrm{N})$ along the $z$-axis is much smaller than the in-plane forces and the required levitation force, pointing downwards. This result confirms that all the levitation force is provided by streaming. The streaming velocity along the $z$-axis in both simulation and PIV measurement are compared in Fig. 4(d), where good agreement in trend can be found despite non-negligible noise in PIV measurements. As a comparison, we have also measured 
the free-fall speed of the particle in water tank by tracking the particle position in a video. The measured free-fall speed is $83 \mathrm{~mm} / \mathrm{s}$, which is close to the streaming flow velocity at $\mathrm{z}=42 \mathrm{~mm}$ from simulation and PIV measurement. The small discrepancy is due to the slight change in field distribution, the existence of PIV particles, and that the viscosity of water changes over time due to the dissolved gas, dust. Such a result also confirms that the required levitation force is providing by acoustic streaming. The stiffness of the trap $(k)$ was calculated as the gradient of the force [18]. From Fig. 4(b), it can be calculated that the lateral stiffness is $k_{x}=0.52 \mathrm{mN} / \mathrm{m}$. Since the PIV measurement is noisy, the vertical stiffness is estimated by fitting the data with a rational curve, and calculate the stiffness of the curve, resulting in $k_{z}=0.064 \mathrm{mN} / \mathrm{m}$. It is worth noting that, since the levitation is provided by the drag force in flow instead of radiation force, the upward levitation force is less sensitive to the shape and material properties of the particle. As a demonstration, we have also shown the trapping of cylindrical particles with diameter $1.3 \mathrm{~mm}$ and height $1.3 \mathrm{~mm}$, and total weight $6 \mathrm{mg}$, as shown in mov. S2 and mov. S3. In this case, the calculated levitation force reaches $41.8 \mu \mathrm{N}$. As a comparison, we dropped another particle outside the focused region, and it sinks quickly, as is shown in mov. S3. Note that the applied voltage on the transducer can be further increased for faster streaming, while the first-order linear field shape is preserved. Therefore, the tweezer can lift the heavier particle without sacrificing its in-plane trapping capability. We also demonstrate the ability to move the particle along a prescribed three dimensional trajectory by scanning the source transducer, as shown in the inset of Fig. 4 and mov. S4, where the trapped particle closely follows the designed path.

The hybrid acoustic tweezer relies on the balance of drag force and gravity, Therefore, it is designed to work in the upward orientation. Nevertheless, the trap stays stable even when the setup is tilted. From the experimental results shown in mov. S5, we can see that the tweezer can stably trap the particle at a tilting angle as large as $21^{\circ}$.

Compared with the 3D trap demonstrated by Baresch et al. [31] where the trapping force along $z$ direction relies on the dipole mode in a sphere, levitating particles with drag in the streaming flow offers several advantages. First, it is able to levitate heavier particles since the drag in streaming flow can provide larger upward force than using radiation forces. Second, it removes the dependence of trapping on the shape and material properties of the levitated particle. Third, the wave field is generated by a single transducer and passive lens instead of the transducer array, which provides an inexpensive and reliable route for contact-free particle and fluid manipulation.

\section{Discussion}

Radiation force acoustic tweezers are a versatile platform for object manipulation capable of handling a wide range of applications in biology, chemistry, and medicine, owning to their simplicity and biocompatibility. However, for particle manipulation in the three dimensional space, there is a trade off between particle parameters and acoustic amplitude because of the stability issue and nonlinear streaming perturbation induced by finite amplitude acoustic waves. Here we have described and demonstrated a hybrid 3D single beam acoustic tweezer by combining the radiation force and acoustic streaming. We show that instead of being a nuisance, carefully designed acoustic streaming can be harnessed to help control particles in fluids and create a fully 3D trap. As a proof of concept, we have (i) designed a focused acoustic vortex lens that facilities acoustic vortex trapping and localized upward streaming flow simultaneously, (ii) verified the designed acoustic field and the corresponding streaming field in both simulation and experiments, and (iii) demonstrated three dimensional trapping and manipulation of particles in fluid.

Compared with previously reported 3D acoustic tweezers in fluids, using acoustic streaming to defy gravity provides several benefits. First, since streaming tweezers rely on streaming drag instead of radiation force, they can accommodate a broader range of material properties and particle shapes, especially for larger and heavier particle cases where radiation force is not sufficient to lift the particle. For example, in our case, the levitation force provided by streaming is about 3 orders of magnitude larger than previously reported radiation force tweezers, when scaled to the same operating frequency. Second, the levitation force can be tunable, so the same tweezer can adapt to a wide range of particle materials by simply tuning the ultrasound amplitude, and thus offers great versatility. Third, for biomedical applications where trapped particles are small, radiation force tweezers require scaling up the frequency to keep a radius-to-wavelength ratio sufficiently large. However, sound absorption and non-linearity effects become more significant for higher frequencies, so that streaming becomes unavoidable, making radiation tweezers less reliable. In contrast, streaming tweezers are expected to take advantage of high frequencies since they naturally appear. For example, stem cell differentiation and migration has been proven to be highly dependent on the strain and fluid flow. They have significant impact on bone regeneration, tissue growth, and embryonic development [41-43]. Such studies are typically conducted in microfluidic chambers. A non-contact device that hold the cell and exert flow on it simultaneously may lead to interesting studies on cell differentiation. Fourth, for the radiation force tweezers, interference between incident wave and reflected waves from the boundary may affect the trap. However, since streaming field is highly localized, changing the far-field boundaries is not expected to dramatically change the flow dynamics around the trapping point. 
There are also several limitations to the proposed tweezers. First, since the streaming flow velocity is not dropping drastically due to the inertia of water, the trapping stiffness in the axial direction will be lower than the radial direction. This is a common issue for single-sided three dimensional acoustic tweezers, and how to improve its axial resolution remains an open question. Second, the size of the trapped particle cannot be larger than the acoustic and streaming vortex. Otherwise the scattering from the particle may affect the formation of streaming flow. For small particles, streaming will have larger influence on the particle behavior than radiation forces, which may affect the stability in $\mathrm{x}-\mathrm{y}$ plane. In this case, an acoustic tweezer with higher frequency is suggested. Third, the trap relies on the balance between streaming and gravity, so that it is expected to work only if it is orientated upwards. Fourth, due to the nonlinear nature of acoustic streaming, the relation between the required levitation force and applied acoustic amplitude is not linear. In addition, the precise characterization of the levitation force requires coupling between the nonlinear acoustic field, fluid mechanics, and fluid-structure interaction, which adds difficulty for analytical calculation and full simulation. Therefore, in the current setup, adaption to various particles is achieved by careful tuning of the input amplitude. Other possible ways to tune the levitation force may include applying short pulsed signals with different duty cycle [27]. Nevertheless, we have shown that streaming offers an additional degree of freedom for acoustic tweezers that can greatly extend its versatility.

\section{Methods}

\section{Fabrication of the lens}

The lens is fabricated with the standard PDMS molding process. A negative mold was fabricated with stereolithography 3D Printing. Part A and part B of Ecoflex 00-30 Silicone is mixed thoroughly by 1:1 weight ratio, degassed with a vacuum chamber and then poured into the mold. The mold is then baked in an oven at 120 Celsius degrees for an hour for the silicone to cure. The lens is then separated from the mold and attached to the piezoelectric patch.

\section{Numerical simulations}

The material thickness profile for the PDMS lens is calculated from the phase map for the holographic element [17]:

$$
T(r, \theta)=T_{0}-\frac{\Delta \phi(r, \theta)}{k_{m}-k_{h}}
$$

where $T(r, \theta)$ is the thickness of the lens pixel positioned at point with polar coordinates $(r, \theta), T_{0}$ is the initial baseline thickness, $k_{h}$ and $k_{m}$ are the wave numbers in the hologram fabrication material and its surrounding medium, and $\Delta \phi(r, \theta)$ in our case is the phase map of the focused vortex sound wave source, calculated from the equation:

$$
\Delta \phi(r, \theta)=k_{m}\left(\sqrt{r^{2}+f^{2}}-f\right)+\theta
$$

where $(r, \theta)$ are the pixel polar coordinates and $f$ is the focal distance of the lens. The calculated thickness profile is then used to construct the 3D solid model (in STEP format) of the lens using CAD software Salome 8.3 [44, 45] with Python 2.7 interface. The STEP solid model of the lens is then ready for 3D printing.

The acoustic finite element simulations were performed in Salome-MECA 2017 CAE environment under Ubuntu 16 LTS system, using Code-Aster 11.3 solver [38] for the numerical calculations of pressure acoustics in frequency domain and ParaVis plugin for post-processing. The simulations of acoustic streaming were performed in OpenFOAM v6 finite volume tool [39] in two stages, using a slightly modified compressible flow sonicLiquidFoam solver for the acoustic field simulation in time domain, and icoFoam, incompressible steady-state solver for the simulation of the streaming fluid flow itself. The streaming flow effect is achieved by adding an external force term into the second stage incompressible flow equations [46]:

$$
\mathbf{F}=\left\langle-\rho \frac{\partial \mathbf{u}}{\partial t}-\rho_{0}(\mathbf{u} \cdot \nabla) \mathbf{u}\right\rangle
$$

where $\mathbf{u}$ is the acoustic particle velocity and $\rho$ is the compressible fluid density calculated during the first acoustic simulation stage, $\rho_{0}$ is the equilibrium constant density, and $\langle\cdot\rangle$ indicates the time-averaging of the term over a significant number of iterations. The dynamic viscosity of water is set as $\mu=0.0044 \mathrm{~Pa} \cdot \mathrm{s}$, calculated from the free-fall speed of the particle using Stokes ${ }^{6}$ Law. Note that a more precise modeling of acoustic streaming has been developed recently $[47,48]$. In this study, we seek to qualitatively predict the flow distribution with simulation, therefore, a simpler form is adopted. Even with such a simple form, we can still see a reasonable agreement between simulation and experiments. 


\section{Calculation of radiation forces}

The radiation force on the particle is calculated semi-analytically. First, the entire acoustic pressure and velocity field is calculated using Angular Spectrum Analysis (ASA) method [49]. Then for a specific particle, the Gor'kov potential field $U$ can be calculated as [50]:

$$
U=2 \pi R^{3}\left(\frac{\left\langle p^{2}\right\rangle}{3 \rho_{0} c_{0}^{2}} f_{1}-\frac{\rho_{0}\left\langle v^{2}\right\rangle}{2} f_{2}\right)
$$

where $f_{1}=1-\frac{\rho_{0} c_{0}^{2}}{\rho_{p} c_{p}^{2}}, f_{2}=\frac{2\left(\rho_{p}-\rho_{0}\right)}{2 \rho_{p}+\rho_{0}}$ are constants that characterize monopole and dipole responses, respectively. The subscript $\mathrm{p}$ denotes the particle. The radiation force can then be estimated using $F=-\nabla U$. Note here that here we used simple model to calculate the radiation forces. More precise models have been developed to take into account the shear modulus of the particle [31].

\section{Acoustic field measurement and Particle imaging velocimetry}

The measurement was performed in a 40-gallon water tank. For the measurement of the linear acoustic field, the computer-controlled function generator (RIGOL DG4102) generates a Gaussian-modulated pulse centered at $500 \mathrm{kHz}$. The signal is amplified by ENI 2100L RF power amplifier and drives the PZT disk. A hydrophone (ONDA HNR-0500) is attached to a 3D scanning stage to scan the field. The output signal from the hydrophone is recorded by AlazarTech ATS 9440 waveform digitizer at a sample rate $125 \mathrm{MS} / \mathrm{s}$. The signal at each scanned position is averaged over 1024 measurements to eliminate noises. Then Fourier transform is performed to extract the amplitude and phase for 500 $\mathrm{kHz}$ to generate the field map.

For the measurement of the flow field, we adopted particle image velocimetry (PIV). Polyamide seeding particles with density $1.03 \mathrm{~g} / \mathrm{cm}^{3}$ and mean size $60 \mu \mathrm{m}$ are dispersed in water. A $532 \mathrm{~nm}$ laser line generator emits a fan-shaped beam and the light plane is aligned with $x-z$ plane. The flow is recorded with the slow motion mode of a cellphone camera. The video is processed with PIVlab, an open source toolbox in MATLAB. Since the particle is sparsely dispersed in the fluid, the ensemble correlation PIV algorithm is adopted, where 2000 frames are used as an ensemble.

\section{Data and materials}

All data needed to evaluate the conclusions in the paper are presented in the paper. Additional data related to this paper may be requested from the authors.

\section{References}

[1] D. Baresch, J.-L. Thomas, and R. Marchiano, The Journal of the Acoustical Society of America 133, 25 (2013).

[2] A. Ozcelik, J. Rufo, F. Guo, Y. Gu, P. Li, J. Lata, and T. J. Huang, Nature methods p. 1 (2018).

[3] J. Shi, D. Ahmed, X. Mao, S.-C. S. Lin, A. Lawit, and T. J. Huang, Lab on a Chip 9, 2890 (2009).

[4] S. Tran, P. Marmottant, and P. Thibault, Applied Physics Letters 101, 114103 (2012).

[5] X. Ding, S.-C. S. Lin, B. Kiraly, H. Yue, S. Li, I.-K. Chiang, J. Shi, S. J. Benkovic, and T. J. Huang, Proceedings of the National Academy of Sciences 109, 11105 (2012).

[6] D. Foresti, M. Nabavi, M. Klingauf, A. Ferrari, and D. Poulikakos, Proceedings of the National Academy of Sciences 110, 12549 (2013).

[7] X. Ding, Z. Peng, S.-C. S. Lin, M. Geri, S. Li, P. Li, Y. Chen, M. Dao, S. Suresh, and T. J. Huang, Proceedings of the National Academy of Sciences 111, 12992 (2014).

[8] D. Foresti and D. Poulikakos, Physical review letters 112, 024301 (2014).

[9] D. J. Collins, B. Morahan, J. Garcia-Bustos, C. Doerig, M. Plebanski, and A. Neild, Nature communications 6, 8686 (2015).

[10] P. Augustsson, J. T. Karlsen, H.-W. Su, H. Bruus, and J. Voldman, Nature communications 7, 11556 (2016).

[11] D. J. Collins, C. Devendran, Z. Ma, J. W. Ng, A. Neild, and Y. Ai, Science advances 2, e1600089 (2016). 
[12] F. Guo, Z. Mao, Y. Chen, Z. Xie, J. P. Lata, P. Li, L. Ren, J. Liu, J. Yang, M. Dao, et al., Proceedings of the National Academy of Sciences 113, 1522 (2016).

[13] J. W. Ng, C. Devendran, and A. Neild, Lab on a Chip 17, 3489 (2017).

[14] Z. Tian, S. Yang, P.-H. Huang, Z. Wang, P. Zhang, Y. Gu, H. Bachman, C. Chen, M. Wu, Y. Xie, et al., Science advances 5, eaau6062 (2019).

[15] C. E. Démoré, P. M. Dahl, Z. Yang, P. Glynne-Jones, A. Melzer, S. Cochran, M. P. MacDonald, and G. C. Spalding, Physical review letters 112, 174302 (2014).

[16] A. Marzo, S. A. Seah, B. W. Drinkwater, D. R. Sahoo, B. Long, and S. Subramanian, Nature communications 6, 8661 (2015).

[17] K. Melde, A. G. Mark, T. Qiu, and P. Fischer, Nature 537, 518 (2016).

[18] A. Franklin, A. Marzo, R. Malkin, and B. Drinkwater, Applied Physics Letters 111, 094101 (2017).

[19] G. Memoli, M. Caleap, M. Asakawa, D. R. Sahoo, B. W. Drinkwater, and S. Subramanian, Nature communications 8, 1 (2017).

[20] M. Prisbrey and B. Raeymaekers, Physical Review Applied 10, 034066 (2018).

[21] A. Marzo and B. W. Drinkwater, Proceedings of the National Academy of Sciences 116, 84 (2019).

[22] B. T. Hefner and P. L. Marston, The Journal of the Acoustical Society of America 106, 3313 (1999).

[23] D. Baresch, J.-L. Thomas, and R. Marchiano, Journal of Applied Physics 113, 184901 (2013).

[24] C. R. Courtney, C. E. Demore, H. Wu, A. Grinenko, P. D. Wilcox, S. Cochran, and B. W. Drinkwater, Applied Physics Letters 104, 154103 (2014).

[25] X. Jiang, Y. Li, B. Liang, J.-c. Cheng, and L. Zhang, Physical review letters 117, 034301 (2016).

[26] A. Riaud, M. Baudoin, O. B. Matar, L. Becerra, and J.-L. Thomas, Physical Review Applied 7, 024007 (2017).

[27] A. Marzo, M. Caleap, and B. W. Drinkwater, Physical review letters 120, 044301 (2018).

[28] M. Baudoin, J.-C. Gerbedoen, A. Riaud, O. B. Matar, N. Smagin, and J.-L. Thomas, Science advances 5, eaav1967 (2019).

[29] L. Zhang and P. L. Marston, The Journal of the Acoustical Society of America 129, 1679 (2011).

[30] L. Zhang and P. L. Marston, Physical Review E 84, 065601 (2011).

[31] D. Baresch, J.-L. Thomas, and R. Marchiano, Physical review letters 116, 024301 (2016).

[32] J. Friend and L. Y. Yeo, Reviews of Modern Physics 83, 647 (2011).

[33] D. Ahmed, A. Ozcelik, N. Bojanala, N. Nama, A. Upadhyay, Y. Chen, W. Hanna-Rose, and T. J. Huang, Nature communications 7, 11085 (2016).

[34] A. Hashmi, G. Yu, M. Reilly-Collette, G. Heiman, and J. Xu, Lab on a Chip 12, 4216 (2012).

[35] P.-H. Huang, N. Nama, Z. Mao, P. Li, J. Rufo, Y. Chen, Y. Xie, C.-H. Wei, L. Wang, and T. J. Huang, Lab on a Chip 14, 4319 (2014).

[36] H. Van Phan, M. B. Coşkun, M. Şeşen, G. Pandraud, A. Neild, and T. Alan, Lab on a Chip 15, 4206 (2015).

[37] S. P. Zhang, J. Lata, C. Chen, J. Mai, F. Guo, Z. Tian, L. Ren, Z. Mao, P.-H. Huang, P. Li, et al., Nature communications 9, 2928 (2018).

[38] EDF, Finite element code_aster, analysis of structures and thermomechanics for studies and research, Open source on www.code-aster.org (1989-2017).

[39] Open source on www.openfoam.org.

[40] S. O. Catarino, J. M. Miranda, S. Lanceros-Mendez, and G. Minas, The Canadian Journal of Chemical Engineering 92 (2014).

[41] D. J. Kelly and P. Prendergast, Journal of biomechanics 38, 1413 (2005).

[42] H. Huang, Y. Nakayama, K. Qin, K. Yamamoto, J. Ando, J. Yamashita, H. Itoh, K. Kanda, H. Yaku, Y. Okamoto, et al., Journal of Artificial Organs 8, 110 (2005).

[43] W. J. Polacheck, J. L. Charest, and R. D. Kamm, Proceedings of the National Academy of Sciences 108, 11115 (2011).

[44] A. Ribes and C. Caremoli, COMPSAC 07: Proceeding of the 31st Annual International Computer Software and Applications Conference pp. 553-564 (2007).

[45] Open source on www.salome-platform.org.

[46] P. B. Muller, R. Barnkob, M. J. H. Jensen, and H. Bruus, Lab on a Chip 12, 4617 (2012).

[47] A. Riaud, M. Baudoin, O. B. Matar, J.-L. Thomas, and P. Brunet, Journal of Fluid Mechanics 821, 384 (2017).

[48] M. Baudoin and J.-L. Thomas, Annual Review of Fluid Mechanics 52 (2019).

[49] J. W. Goodman, Introduction to Fourier optics (Roberts and Company Publishers, 2005).

[50] L. Gor'kov, Soviet Physics Doklady 6, 773 (1962).

\section{Acknowledgments}

This work was supported by the Multidisciplinary University Research Initiative grant from the Office of Naval Research (N00014-13-1-0631), an Emerging Frontiers in Research and Innovation grant from the National Science Foundation (Grant No. 1641084). Z.F. and A.C. would like to acknowledge A-STAR for funding support under AMEIRG18-0081. Z.F. want to thank Tan Chin Tuan exchange followship for providing opportunities for collaboration. The authors are grateful for Tony Jun Huang for the helpful discussions. 


\section{Author Contribution}

S.A.C. and Z. F. supervised the project. A. C. developed the mathematical model and performed simulations. J. L., A. C. and Z. F. fabricated the samples, J.L., Z. F. and X. P. realized the experiments. J.L., X. P. and Y. P. processed the experimental data. All authors contributed to discussing the results and preparing the manuscript.

\section{Competing interests}

The Authors declare no Competing Financial or Non-Financial Interests. 
(a)

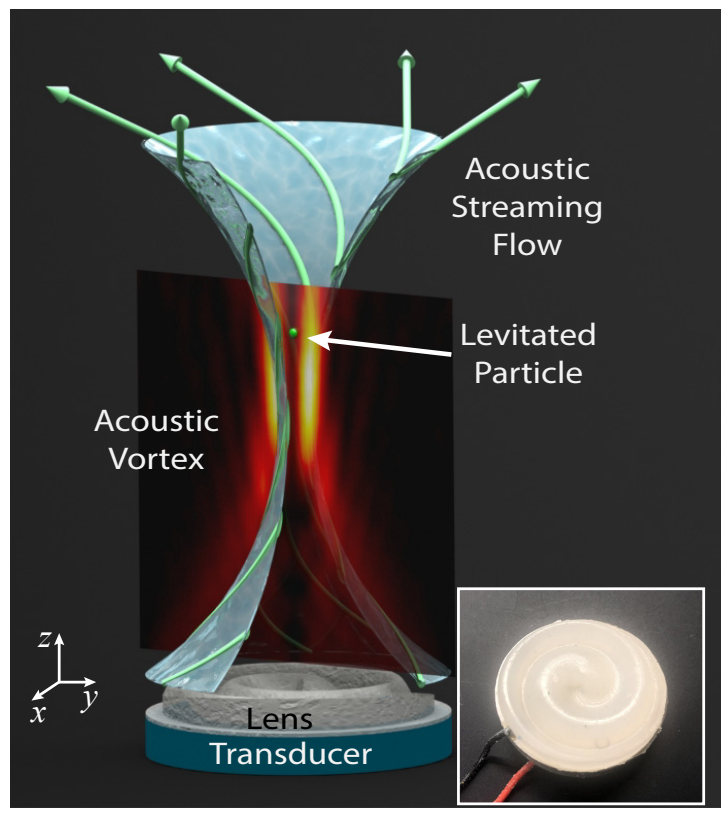

(b)

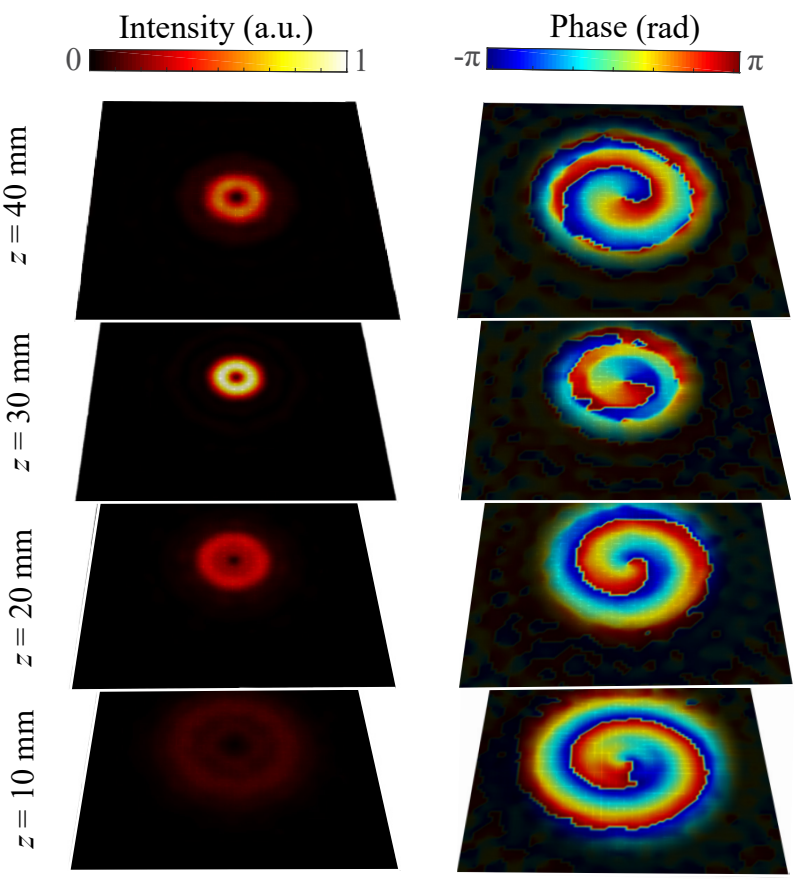

FIG. 1: Schematics of the design and working principle. (a) Creating a focused acoustic vortex for in-plane particle trapping, and the localized gradient streaming field levitates the particle, providing trap in the third dimension.

Inset shows a photo of the fabricated device. (b) Evolution of the intensity and phase fields across different cut planes along $z$ axis. The field is gradually focused as it propagates, keeping the spiral phase profile in the central region. 


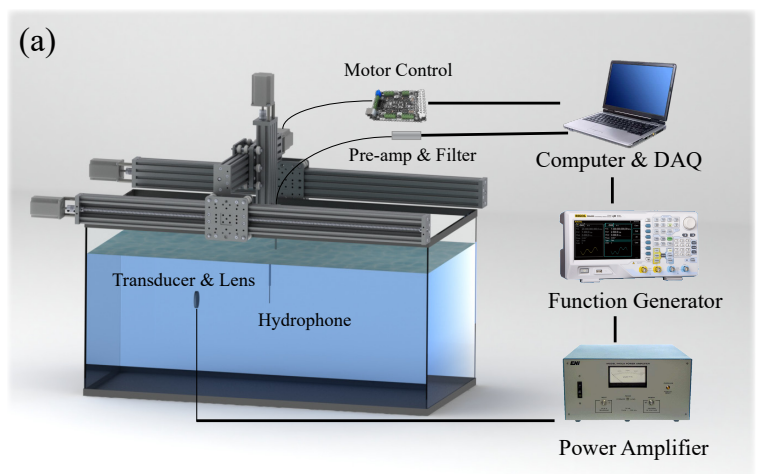

Simulation

(b) Intensity: $x-y$ plane, $z=30 \mathrm{~mm}$

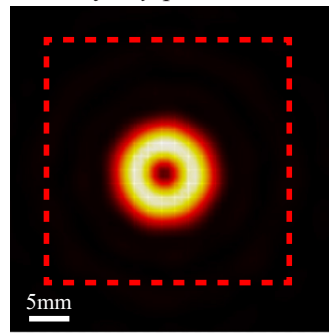

(d)
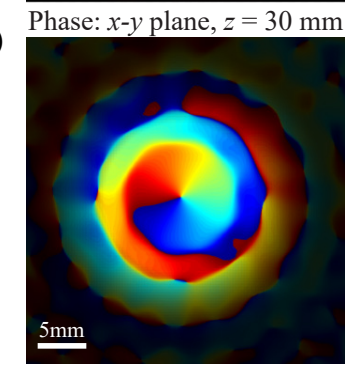

(f)

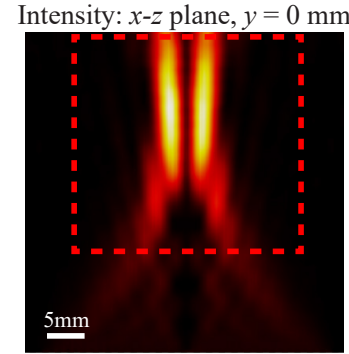

(h)

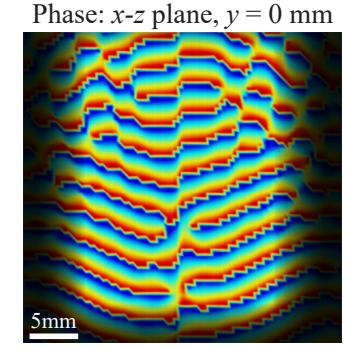

Experiment

(c) Intensity: $x-y$ plane, $z=30 \mathrm{~mm}$

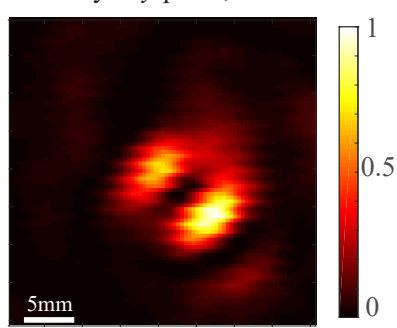

(e)

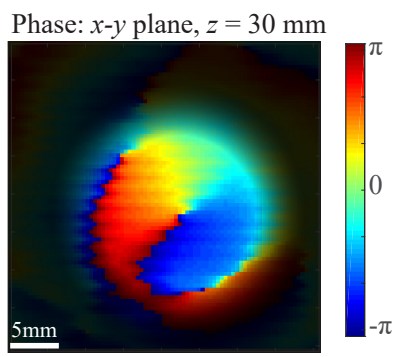

(g) Intensity: $x-z$ plane, $y=0 \mathrm{~mm}$

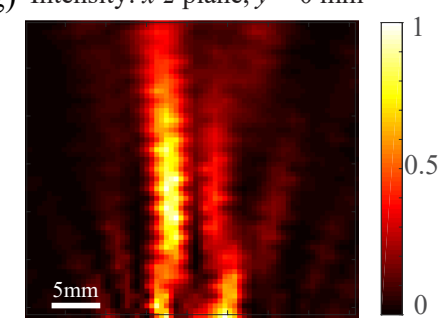

(i) Phase: $x$-z plane, $y=0 \mathrm{~mm}$

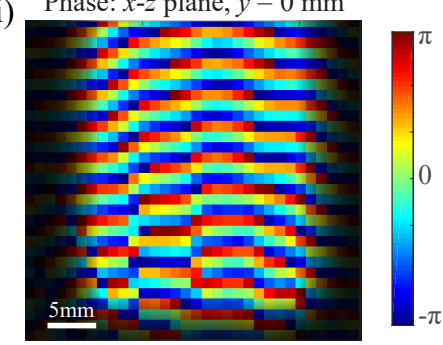

FIG. 2: Measurement of the acoustic field. (a) Illustration of the ultrasound scanning system (b, d) Simulated intensity and phase field of the $x-y$ plane at $z=30 \mathrm{~mm}$. The ring-shaped intensity profile provides the $2 \mathrm{D}$ trap. The spiral phase profile is clearly seen in the central region. (f, h) Simulated intensity and phase field of $x-z$ plane at $y=0$. The intensity forms a node line along $z$ axis, along which the phase is discontinuous. (c, e, g, i) The corresponding measured intensity and phase field in the experiment, in great agreement with the simulation. 


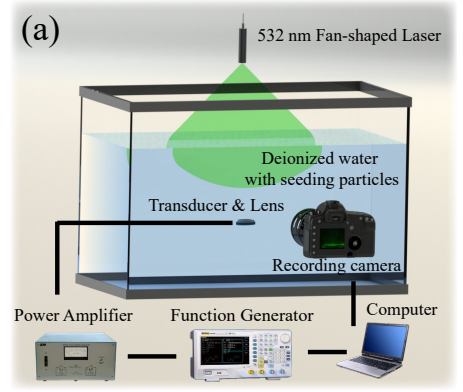

(b) Streaming Velocity Field: Simulation

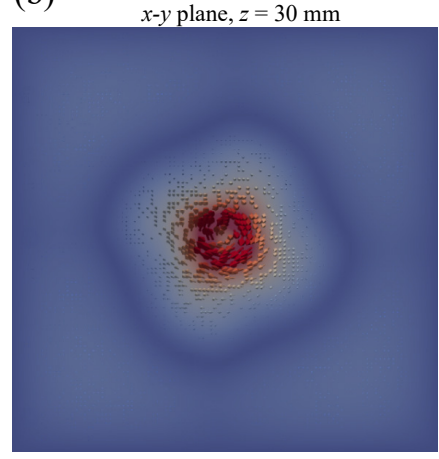

(c)

Streaming Velocity Field: Simulation $x-z$ plane, $y=0 \mathrm{~mm}$

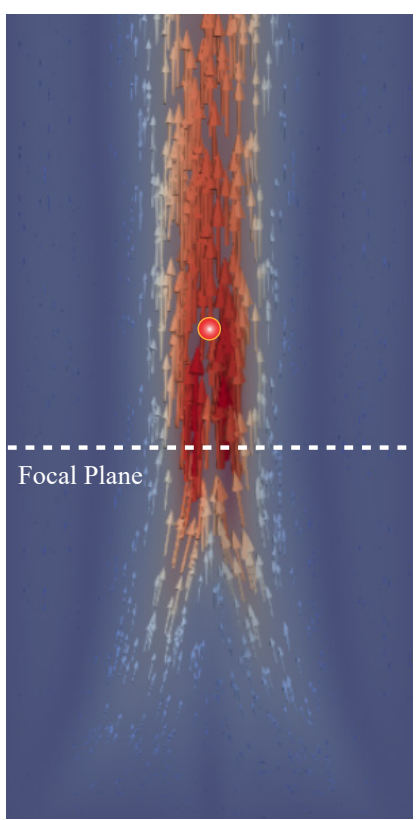

(d) Streaming Velocity Field: Experiment $x-z$ plane, $y=0 \mathrm{~mm}$

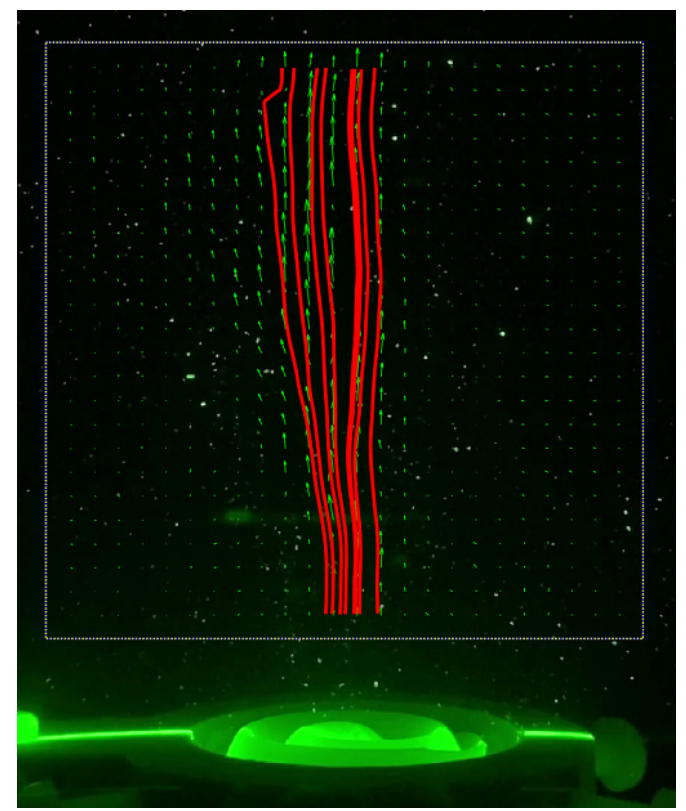

FIG. 3: Streaming field measurement setup and results. (a) Illustration of the streaming field measurement setup. (b) Simulation of streaming velocity field within $x-y$ plane at $z=30 \mathrm{~mm}$. The streaming flow is focused in the central region, forming a streaming vortex (c) Simulation of streaming velocity field along $x$ - $z$ plane at $y=0$ mm. The velocity increases towards the focal point, and gradually decreases after a certain height. The low velocity along the node line is the results of the streaming vortex. The red dot denotes the trapped particle.

(d) Measured streaming velocity field along $x$ - $z$ plane at $y=0 \mathrm{~mm}$, in good agreement with the simulation. 

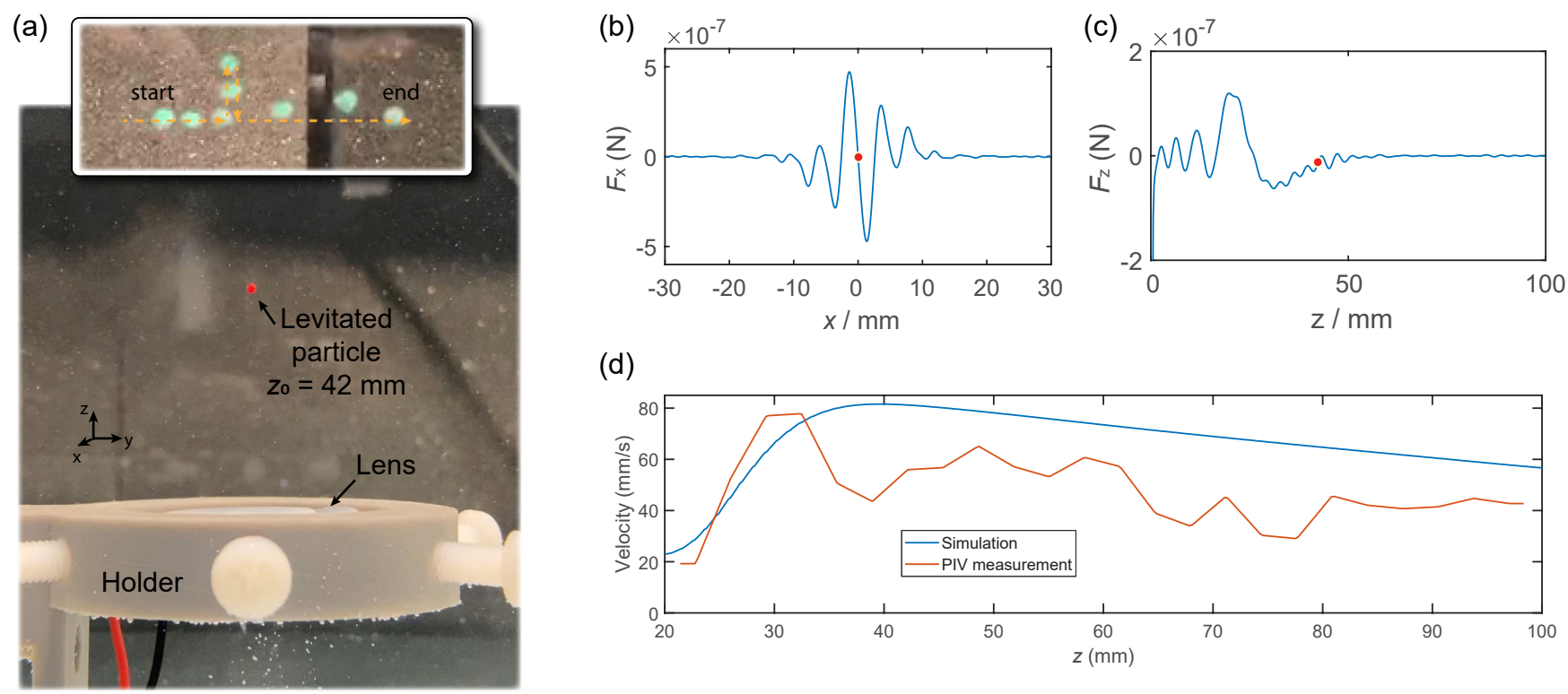

(d)

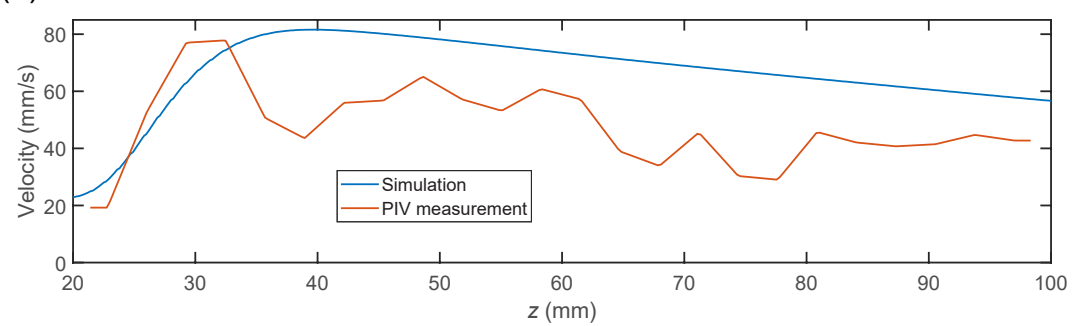

FIG. 4: Snapshot of the levitated particle. (a) The red particle is stably trapped in 3D at $z_{0}=42 \mathrm{~mm}$ (Supplementary movie S1). The inset shows the trace of a particle moving along a designed path outlined by the yellow arrows. The particle closely follows the 3D motion of the lens (Supplementary movie S4). (b) Radiation force along $x$ direction in the plane where particle is trapped. The red dot denotes the particle position. (c) Radiation force along z-axis. (d) streaming velocity along $z$-axis in simulation and PIV measurement, showing a flow velocity gradient that creates the trap along the $z$-axis. 
(a)

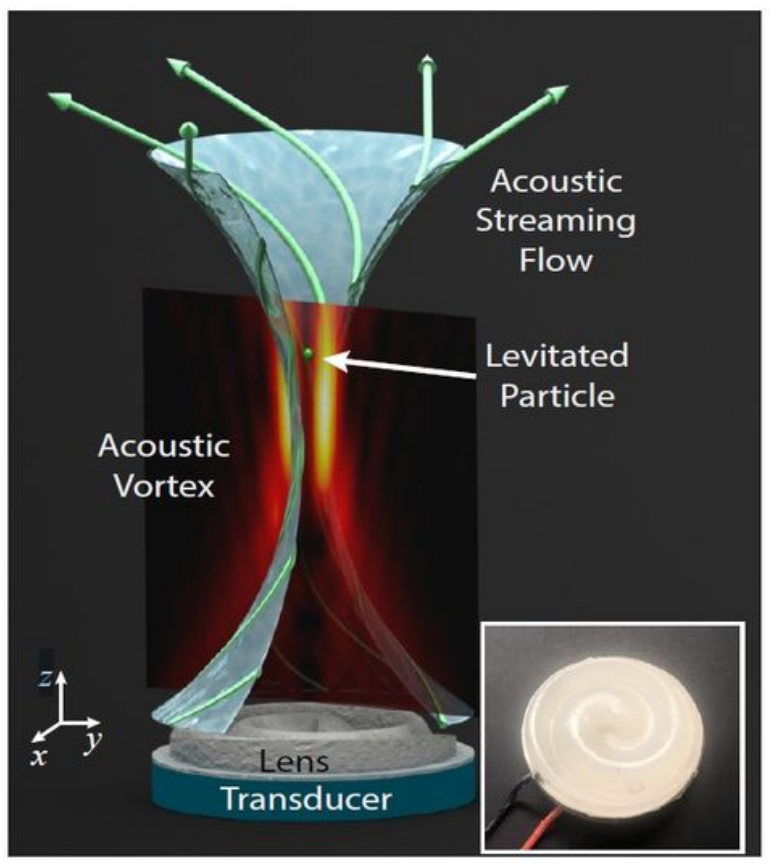

(b)

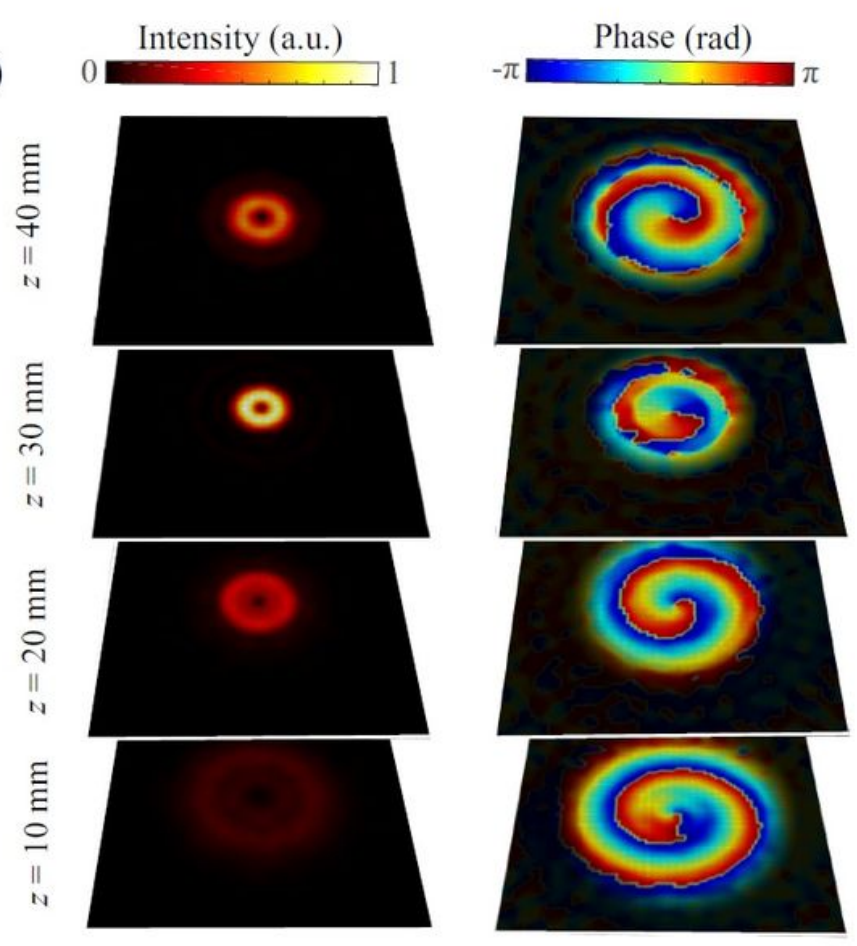

\section{Figure 1}

Schematics of the design and working principle. (a) Creating a focused acoustic vortex for in-plane particle trapping, and the localized gradient streaming field levitates the particle, providing trap in the third dimension. Inset shows a photo of the fabricated device. (b) Evolution of the intensity and phase fields across different cut planes along $z$ axis. The field is gradually focused as it propagates, keeping the spiral phase profile in the central region. 


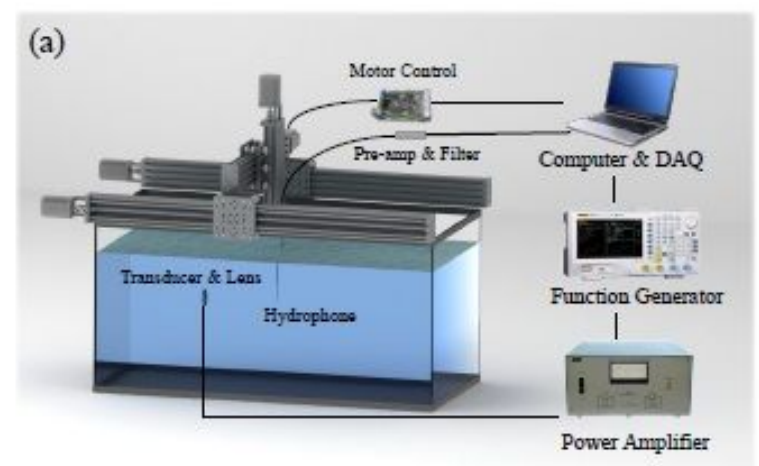

Simulation

(b) Intensity: $x-y$ plane, $z=30 \mathrm{~mm}$

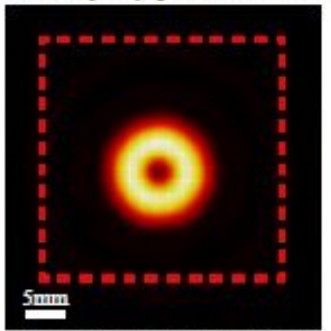

(d)

Phase: $x-y$ plane, $z=30 \mathrm{~mm}$

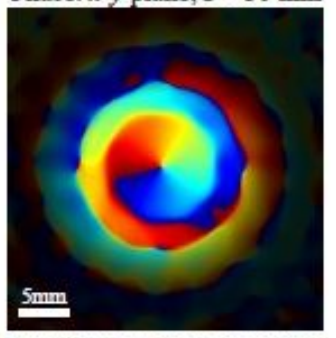

(f)

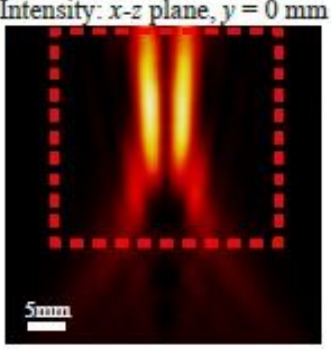

(h) Phase: $x-z$ plane, $y=0 \mathrm{~mm}$

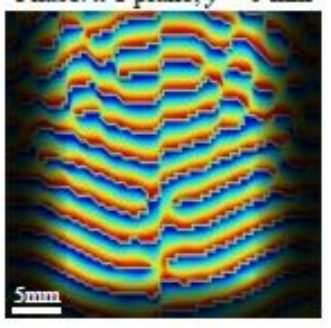

Experiment

(c) Intensity: $x-y$ plane, $z=30 \mathrm{~mm}$

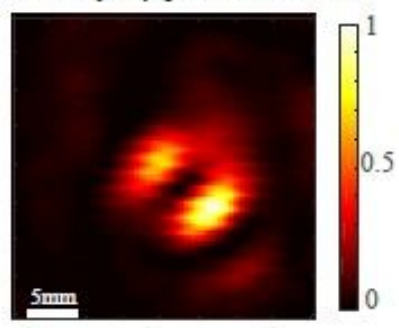

(e) Phase: $x-y$ plane, $z=30 \mathrm{~mm}$

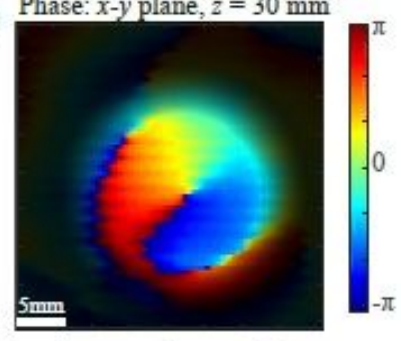

(g) Intensity: $x-z$ plane, $y=0 \mathrm{~mm}$

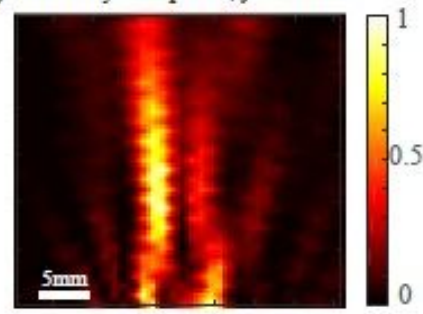

(i) Phase: $x-z$ plane, $y=0 \mathrm{~mm}$

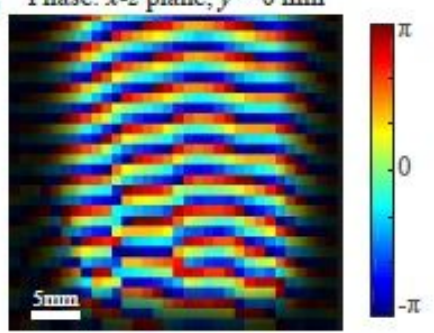

Figure 2

Measurement of the acoustic field. (a) Illustration of the ultrasound scanning system (b, d) Simulated intensity and phase field of the $x-y$ plane at $z=30 \mathrm{~mm}$. The ring-shaped intensity profile provides the 2D trap. The spiral phase profile is clearly seen in the central region. ( $f, h)$ Simulated intensity and phase field of $x-z$ plane at $y=0$. The intensity forms a node line along $z$ axis, along which the phase is 
discontinuous. (c, e, g, i) The corresponding measured intensity and phase field in the experiment, in great agreement with the simulation.

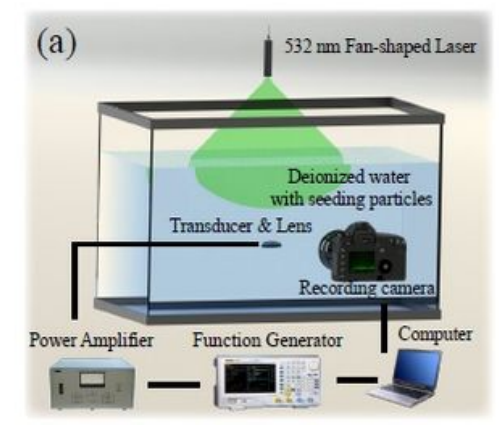

(b) Streaming Velocity Field: Simulation

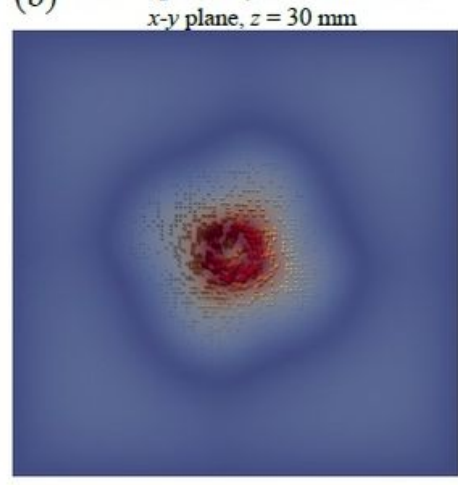

(c) Streaming Velocity Field: Simulation $x-z$ plane, $y=0 \mathrm{~mm}$

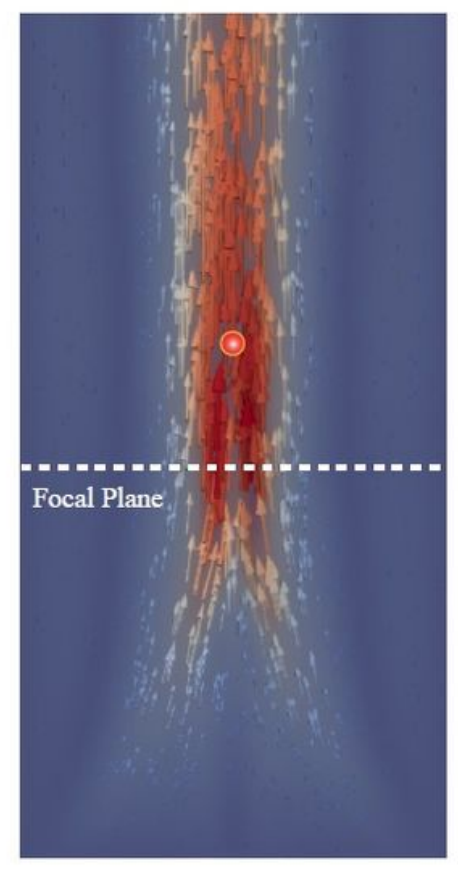

(d) Streaming Velocity Field: Experiment $x-z$ plane, $y=0 \mathrm{~mm}$

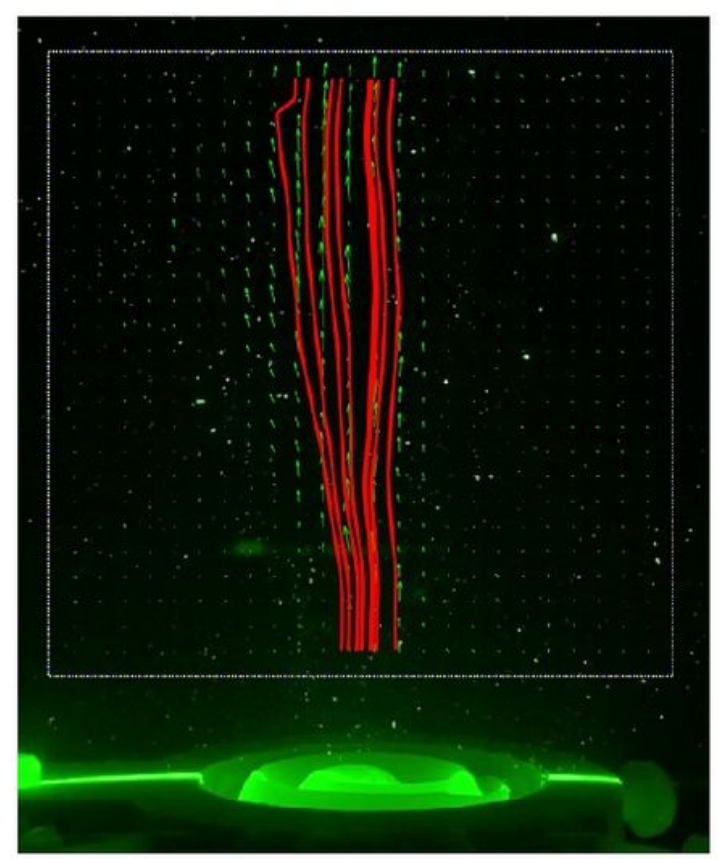

\section{Figure 3}

Streaming field measurement setup and results. (a) Illustration of the streaming field measurement setup. (b) Simulation of streaming velocity field within $x-y$ plane at $z=30 \mathrm{~mm}$. The streaming flow is focused in the central region, forming a streaming vortex (c) Simulation of streaming velocity field along $x-z$ plane at $y=0 \mathrm{~mm}$. The velocity increases towards the focal point, and gradually decreases after a certain height. The low velocity along the node line is the results of the streaming vortex. The red dot denotes the trapped particle. (d) Measured streaming velocity field along $x-z$ plane at $y=0 \mathrm{~mm}$, in good agreement with the simulation. 

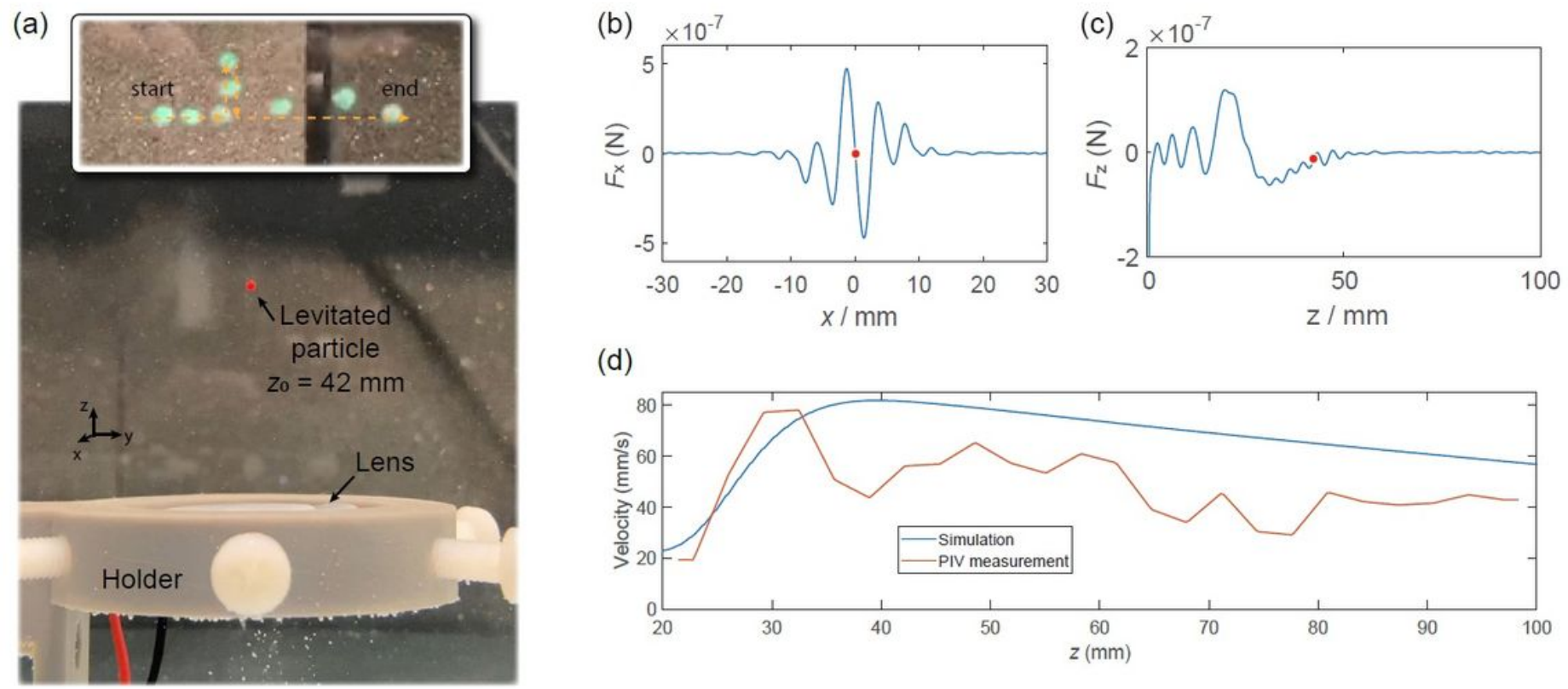

(d)

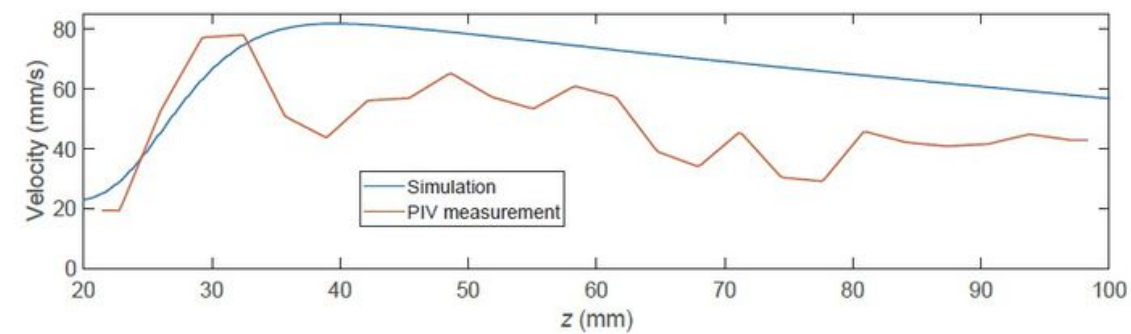

\section{Figure 4}

Snapshot of the levitated particle. (a) The red particle is stably trapped in $3 \mathrm{D}$ at $\mathrm{zO}=42 \mathrm{~mm}$ (Supplementary movie S1). The inset shows the trace of a particle moving along a designed path outlined by the yellow arrows. The particle closely follows the 3D motion of the lens (Supplementary movie S4). (b) Radiation force along $x$ direction in the plane where particle is trapped. The red dot denotes the particle position. (c) Radiation force along z-axis. (d) streaming velocity along z-axis in simulation and PIV measurement, showing a ow velocity gradient that creates the trap along the $z$-axis.

\section{Supplementary Files}

This is a list of supplementary files associated with this preprint. Click to download.

- movS1staticlevitationsphere.mp4

- movS2staticlevitaioncylinder.mp4

- movS3comparison.mp4

- movS43Dmoving.mp4

- movS5tiltedlevitation.mp4 